\title{
บusisersaly
}

\section{Sum-Rate Maximization of NOMA Systems under Imperfect Successive Interference Cancellation}

AbuMahady, I., Mohamed, E. B., Ikki, S., \& Yanikomeroglu, H. (2019). Sum-Rate Maximization of NOMA Systems under Imperfect Successive Interference Cancellation. IEEE Communications Letters, 23(3), $474-477$. [8621012]. https://doi.org/10.1109/LCOMM.2019.2893195

Link to publication record in Ulster University Research Portal

Published in:

IEEE Communications Letters

Publication Status:

Published (in print/issue): 01/03/2019

DOI:

10.1109/LCOMM.2019.2893195

\section{Document Version}

Author Accepted version

\section{General rights}

Copyright for the publications made accessible via Ulster University's Research Portal is retained by the author(s) and / or other copyright owners and it is a condition of accessing these publications that users recognise and abide by the legal requirements associated with these rights.

\section{Take down policy}

The Research Portal is Ulster University's institutional repository that provides access to Ulster's research outputs. Every effort has been made to ensure that content in the Research Portal does not infringe any person's rights, or applicable UK laws. If you discover content in the Research Portal that you believe breaches copyright or violates any law, please contact pure-support@ulster.ac.uk. 


\title{
Sum-Rate Maximization of NOMA Systems under Imperfect Successive Interference Cancellation
}

\author{
Islam Abu Mahady, Ebrahim Bedeer, Salama Ikki, and Halim Yanikomeroglu
}

\begin{abstract}
This work addresses the sum-rate maximization for a downlink non-orthogonal multiple access (NOMA) system in the presence of imperfect successive interference cancellation (SIC). We assume that the NOMA users adopt improper Gaussian signalling (IGS), and hence, derive new expressions of their rates under residual interference from imperfect SIC. We optimize the circularity coefficient of the IGS-based NOMA system to maximize its sum-rate subject to quality-of-service $(\mathrm{QoS})$ requirements. Compared to the NOMA with proper Gaussian signaling (PGS), simulation results show that the IGS-based NOMA system demonstrates considerable sum-rate performance gain under imperfect SIC.
\end{abstract}

Index Terms-Improper Gaussian signalling, non-linear optimization, NOMA, sum-rate.

\section{INTRODUCTION}

Non-orthogonal multiple access (NOMA) proposes the adoption of power/code domain to multiplex signal streams from multiple users together and allow them to transmit simultaneously using the same frequency/time/code resources [1]. One of the advantages of NOMA systems is that when the available resource blocks are assigned to weak channel users, they can still be accessed by other strong channel users, which qualifies NOMA techniques to achieve a higher overall spectral efficiency (SE) [2], [3]. However, NOMA techniques achieve this potential higher SE considering perfect successive interference cancellation (SIC) (see, e.g., [1]-[4], and the references therein). In real scenarios, the assumption of perfect SIC at the receiver might not be practical, since there still remain several serious implementation problems by using SIC, e.g., error propagation and complexity scaling [1]. In [5], a unified framework is presented assuming imperfect SIC, which shows that the performance converges to an error floor at the high signal-to-noise ratio (SNR) region and obtain a zero diversity order. Hence it is of great interest to compensate the impact of imperfect SIC for the NOMA systems.

Recent research works have shown that improper Gaussian signalling (IGS) has the potential over proper (conventional) Gaussian signalling (PGS) to enhance the overall achievable rate of systems that suffer from interference [6]. Compared to the PGS scheme which assumes independent real and imaginary signal components with equal power, the IGS scheme loosens these constraints and introduce a circularity coefficient that enables a more general Gaussian signaling scheme [7].

To the best of the authors' knowledge, there is no existing work in the literature that exploits IGS in an effort to maximize the overall sum-rate of the NOMA system under the practical

I. Abu Mahady and S. Ikki are with Lakehead University, ON, Cananda. Emails:\{iabumah, sikki\}@lakeheadu.ca. E. Bedeer is with Ulster University, UK. Email: e.bedeer.mohamed@ulster.ac.uk, and H. Yanikomeroglu is with Carleton University, ON, Canada. Email: halim@sce.carleton.ca. assumption of imperfect SIC, which motivates us to develop this work. In particular, new closed-form expressions for the users' rate are derived for a downlink two-user NOMA system in the presence of imperfect SIC. Using the derived expressions, an optimization problem is formulated to optimize the circularity coefficient to maximize the overall NOMA sum-rate subject to minimum rate requirements constraints. Simulation results show a considerable sum-rate performance gain when using IGS-based NOMA systems compared with PGS-based NOMA systems.

\section{PRELIMINARY: IMPROPER RANDOM VECTORS}

In this section, preliminary of IGS definitions are presented to ease the understanding of the derivation of information rates of NOMA users.

A complex random variable (RV) is called proper if its pseudo-variance is equal to zero, otherwise it is called improper [8]. For a complex RV $x_{i}$, we use $C_{x_{i}}$ and $\hat{C}_{x_{i}}$ to denote the covariance and pseudo-covariance, respectively. Then for the zero-mean input Gaussian signal $x_{i}, \forall i$, we have $C_{x_{i}}=E\left[x_{i} x_{i}^{*}\right], \hat{C}_{x_{i}}=E\left[x_{i} x_{i}\right]$, and the impropriety degree of $x_{i}$ is given as

$$
\kappa_{x_{i}}=\left|\hat{C}_{x_{i}}\right| / C_{x_{i}}, \quad \forall i
$$

where $0 \leq \kappa_{x_{i}} \leq 1$. If $\kappa_{x_{i}}=0$, we say that $x_{i}$ is proper, and if $\kappa_{x_{i}}=1$, we have maximally improper signal. Note that $C_{x_{i}}$ is nonnegative real number equal to the power value of the transmitted signal, while $\hat{C}_{x_{i}}$ is complex number in general.

\section{System Model And Rate Analysis}

We consider a downlink NOMA system with two users (strong channel user and weak channel user) and a base station. The channel coefficient between the base station and user $i$ is denoted by $h_{i}, \forall i=1,2$, that is modelled as a complex Gaussian RV with zero-mean and variance $\sigma_{h_{i}}^{2}$. The noise at the receivers ends are modelled as zero-mean additive white Gaussian random variable with variances $\sigma_{n}^{2}$. Different from the conventional setup where PGS is assumed, in this work, user's 1 signal $x_{1}$ and user's 2 signal $x_{2}$ are zero-mean complex Gaussian RVs which can be improper. Without loss of generality, it is assumed that $\left|h_{1}\right|^{2}>\left|h_{2}\right|^{2}$, i.e. user 1 is with strong channel gain and user 2 is with weak channel gain.

According to the NOMA principle, the transmit power of the weak user's signal must be greater than that of the strong user, i.e., $P_{2}>P_{1}$. Hence, user 2 decodes directly its signal because the interference inflicted by the user 1 is small and can thus be treated as noise. In contrast, user 1 can decode its own signal after cancelling the weak user's decoded signal through a SIC detector [1]. We assume that SIC process at user's 1 receiver 
is imperfect and the residual interference component due to this imperfection is quantified by a factor $\beta(0 \leq \beta \leq 1)$, where $\beta=0$ referes to perfect SIC and $\beta=1$ refers to the fully imperfect SIC.

The input-output relationship for the two-user SISO system can be expressed as

$$
\begin{aligned}
& y_{1}=\sqrt{P_{1}} h_{1} s_{1}+\beta \sqrt{P_{2}} h_{1} s_{2}+n_{1}, \\
& y_{2}=\sqrt{P_{2}} h_{2} s_{2}+\sqrt{P_{1}} h_{2} s_{1}+n_{2},
\end{aligned}
$$

where $s_{i}$ is $i$ th signal and $n_{i}$ is AWGN at the corresponding receivers.

In the following, we derive the rate expressions for the general case of IGS for for both users, i.e., $x_{1}$ and $x_{2}$ are improper. Let $x_{i}=\sqrt{P_{i}} s_{i}, \forall i=1,2$ are the independent signals for user 1 and 2, respectively, and denote the covariance and pseudocovariance of the transmit signal by

$$
\begin{aligned}
& C_{x_{i}}=P_{i} C_{s_{i}}, \\
& \hat{C}_{x_{i}}=P_{i} \hat{C}_{s_{i}}, \forall i=1,2,
\end{aligned}
$$

where $C_{s_{i}}=E\left[s_{i} s_{i}^{*}\right]$ and $\hat{C}_{s_{i}}=E\left[s_{i} s_{i}\right]$. We assume that $C_{s_{i}}=E\left[s_{i} s_{i}^{*}\right]=1, \forall i=1,2$, i.e., transmit a symbol with a unit power. Next, we derive the rate expressions in terms of circularity coefficient. The covariance and pseudo-covariance of $y_{i}, i=1,2$, can be obtained from (2) and (3) as

$$
\begin{aligned}
& C_{y_{1}}=P_{1}\left|h_{1}\right|^{2}+\beta^{2} P_{2}\left|h_{1}\right|^{2}+\sigma_{1}^{2}, \\
& \hat{C}_{y_{1}}=P_{1} \kappa_{s_{1}} h_{1}^{2}+\beta^{2} P_{2} \kappa_{s_{2}} h_{1}^{2}, \\
& C_{y_{2}}=P_{2}\left|h_{2}\right|^{2}+P_{1}\left|h_{2}\right|^{2}+\sigma_{2}^{2}, \\
& \hat{C}_{y_{2}}=P_{2} \kappa_{s_{2}} h_{2}^{2}+P_{1} \kappa_{s_{1}} h_{2}^{2} .
\end{aligned}
$$

Define the noise and the interference-plus-noise terms in (2) and (3), as $z_{i}, i=1,2$, at each receiver, respectively, where $z_{1}=n_{1}$ and $z_{2}=\sqrt{P_{1}} h_{2} s_{1}+n_{2}$, we get

$C_{z_{1}}=\sigma_{1}^{2}, \hat{C}_{z_{1}}=0, C_{z_{2}}=P_{1}\left|h_{2}\right|^{2}+\sigma_{2}^{2}$, and $\hat{C}_{z_{2}}=P_{1} \kappa_{s_{1}} h_{2}^{2}$.

Following [8], the achievable rate expression for a two-user SISO system is given as [6]

$$
R_{i}=\frac{1}{2} \log _{2}\left(\frac{C_{y_{i}}^{2}-\left|\hat{C}_{y_{i}}\right|^{2}}{C_{z_{i}}^{2}-\left|\hat{C}_{z_{i}}\right|^{2}}\right) .
$$

By substituting (6), (7), and (10) into (11), and assuming without loss of generality $\sigma_{1}^{2}=\sigma_{2}^{2}=\sigma^{2}$, the achievable rate expression for the strong user 1 of a NOMA system, in the case of both users adopt IGS, reduces to

$$
\begin{aligned}
R_{1}\left(\kappa_{s_{1}}, \kappa_{s_{2}}\right)= & \underbrace{\log _{2}\left(1+\frac{P_{1}\left|h_{1}\right|^{2}}{\beta^{2} P_{2}\left|h_{1}\right|^{2}+\sigma^{2}}\right)}_{\text {proper }} \\
& +\underbrace{\frac{1}{2} \log _{2}\left(1-\frac{\left|h_{1}^{2} P_{1} \kappa_{s_{1}}\right|^{2}+\left.\left.\left|\beta^{2} P_{2}^{2}\right| h_{1}\right|^{2} \kappa_{s_{2}}\right|^{2}}{\left(P_{1}\left|h_{1}\right|^{2}+\beta^{2} P_{2}\left|h_{1}\right|^{2}+\sigma^{2}\right)^{2}}\right)}_{\text {Improper }} \\
& -\underbrace{\frac{1}{2} \log _{2}\left(1-\frac{\left.\left.\left|\beta^{2} P_{2}^{2}\right| h_{1}\right|^{2} \kappa_{s_{2}}\right|^{2}}{\left(\beta^{2} P_{2}\left|h_{1}\right|^{2}+\sigma^{2}\right)^{2}}\right)}_{\text {Improper }} .
\end{aligned}
$$

Similarly, by substituting (8), (9), and (10) into (11), the achievable rate expression for the weak user 2 of a NOMA system, in the case of both users use IGS, reduces to

$$
\begin{aligned}
R_{2}\left(\kappa_{s_{1}}, \kappa_{s_{2}}\right)= & \underbrace{\log _{2}\left(1+\frac{P_{2}\left|h_{2}\right|^{2}}{P_{1}\left|h_{2}\right|^{2}+\sigma^{2}}\right)}_{\text {Proper }} \\
& +\underbrace{\frac{1}{2} \log _{2}\left(1-\frac{\left|P_{2} h_{2}^{2} \kappa_{s_{2}}\right|^{2}+\left|P_{1} h_{2}^{2} \kappa_{s_{1}}\right|^{2}}{\left(P_{2}\left|h_{2}\right|^{2}+P_{1}\left|h_{2}\right|^{2}+\sigma^{2}\right)^{2}}\right)}_{\text {Improper }} \\
& -\underbrace{\frac{1}{2} \log _{2}\left(1-\frac{\left|P_{1} h_{2}^{2} \kappa_{s_{1}}\right|^{2}}{\left(P_{1}\left|h_{2}\right|^{2}+\sigma^{2}\right)^{2}}\right)}_{\text {Improper }} .
\end{aligned}
$$

Please note that each $R_{i}, i=1,2$, in (12), (13) includes two parts; proper and improper. Substituting $\kappa_{s_{i}}=0, \forall i=1,2$ and $\beta=0$ reduces to the rates of PGS case in perfect SIC, which proves the correctness of the derived expressions.

\section{Optimization PRoblem}

In this section, an optimization problem is formulated to optimize the IGS circularity coefficient in order to maximize the sum-rate of a two-user SISO NOMA system subject to minimum rate requirements of each user. Due to space limitations, we focus on the case where we use IGS for strong user (i.e., $x_{1}$ is improper and $\kappa_{s_{1}} \neq 0$ ) and PGS for weak user (i.e., $x_{2}$ is proper and $\kappa_{s_{2}}=0$ ). Other cases will be investigated in future work. Also, we assume the powers $P_{1}$ and $P_{2}$ are already allocated to user 1 and 2, respectively (i.e., they are not optimization variables). That said, the optimization problem for maximizing the sum-rate under QoS constraints can be formulated as

$$
\begin{array}{cl}
\underset{\kappa_{s_{1}}}{\operatorname{maximize}} & R_{1}\left(\kappa_{s_{1}}\right)+R_{2}\left(\kappa_{s_{1}}\right) \\
\text { subject to } & \mathcal{C} 1: R_{1}\left(\kappa_{s_{1}}\right) \geq R_{\text {min }_{1}}, \\
& \mathcal{C} 2: R_{2}\left(\kappa_{s_{1}}\right) \geq R_{\text {min }_{2}}, \\
& \mathcal{C} 3: 0 \leq \kappa_{s_{1}} \leq 1,
\end{array}
$$

where $R_{1}\left(\kappa_{s_{1}}\right)$ and $R_{2}\left(\kappa_{s_{1}}\right)$ are obtained from (12) and (13), respectively, at $\kappa_{s_{2}}=0 . R_{\min _{1}}$ and $R_{\min _{2}}$ are the minimum rate requirements of the strong user and the weak user, respectively. The constraint $\mathcal{C} 3$ reflects that the circulatory coefficient is between 0 and 1 as shown in Definition 2.

The optimization problem in (14) can be solved by applying the Karush-Kuhn-Tucker (KKT) conditions; however, it is worthy to mention that the obtained circularity coefficient $\kappa_{s_{1}}^{*}$ will be sub-optimal as the the problem in (14) is non-convex. The Lagrangian function can be expressed as

$$
\begin{aligned}
& L\left(\kappa_{s_{1}}, \lambda_{1}, \lambda_{2}\right)=-\left(R_{1}\left(\kappa_{s_{1}}\right)+R_{2}\left(\kappa_{s_{1}}\right)\right) \\
& \quad+\lambda_{1}\left(R_{m_{m_{1}}}-R_{1}\left(\kappa_{s_{1}}\right)\right)+\lambda_{2}\left(R_{m_{i n}}-R_{2}\left(\kappa_{s_{1}}\right)\right),
\end{aligned}
$$

where $\lambda_{1}$ and $\lambda_{2}$ are the non-negative Lagrange multipliers associated with the QoS constraints of user 1 and 2, respectively. The circularity coefficient constraint not considered in 


$$
\begin{aligned}
\kappa_{s_{1}}^{2}=0.5((\Phi+\Psi)+(\Phi-\Psi) & \left.\left(\frac{1+\lambda_{2}}{1+\lambda_{1}}\right)\right) \\
& -0.5\left(\left[(\Phi-\Psi)^{2}\left(1+\left(\frac{1+\lambda_{2}}{1+\lambda_{1}}\right)^{2}\right)+(\Phi-\Psi)\left(\frac{1+\lambda_{2}}{1+\lambda_{1}}\right)(2(\Phi+\Psi)-4 \Omega)\right]\right)^{\frac{1}{2}} .
\end{aligned}
$$

the Lagrangian function will be satisfied later. That said, the KKT conditions can be written as follows [9]

$$
\begin{aligned}
\frac{\partial L\left(\kappa_{s_{1}}^{*}, \lambda_{1}, \lambda_{2}\right)}{\partial \kappa_{s_{1}}} & =0, \\
\lambda_{1}\left(R_{\text {min }_{1}}-R_{1}\left(\kappa_{s_{1}}^{*}\right)\right) & =0, \\
\lambda_{2}\left(R_{\text {min }_{2}}-R_{2}\left(\kappa_{s_{1}}^{*}\right)\right) & =0, \\
R_{\text {min }_{1}}-R_{1}\left(\kappa_{s_{1}}^{*}\right) & \leq 0, \\
R_{\text {min }_{2}}-R_{2}\left(\kappa_{s_{1}^{*}}\right) & \leq 0, \\
\lambda_{1}, \lambda_{2} & \geq 0 .
\end{aligned}
$$

From (16), we can obtain the circularity coefficient $\kappa_{s_{1}}^{*}$ as in (23) on top of this page, where $\Phi=\left(1+\frac{\sigma^{2}}{P_{1}\left|h_{2}\right|^{2}}\right)^{2}, \Psi=$ $\left(1+\frac{P_{2}}{P_{1}}+\frac{\sigma^{2}}{P_{1}\left|h_{2}\right|^{2}}\right)^{2}$, and $\Omega=\left(1+\beta^{2} \frac{P_{2}}{P_{1}}+\frac{\sigma^{2}}{P_{1}\left|h_{1}\right|^{2}}\right)^{2}$. To consider $\mathcal{C} 3$, we need to guarantee that the term under the square root in (23) is positive and also the first term of (23) is greater than the second term of it. The values of $\lambda_{1}$ and $\lambda_{2}$ in (23) can be computed using the subgradient method [9] as follows.

$$
\lambda_{i}^{l+1}=\left[\lambda_{i}^{l}-\alpha_{i}^{l}\left(R_{i}-R_{\text {min }_{i}}\right)\right]^{+}, \quad \forall i=1,2,
$$

where [.] $]^{+}$is defined as $\max (., 0)$ and $\alpha_{i}$ is a sufficiently small step size chosen to equals $0.1 / \sqrt{l}$ where $l$ is the iteration number [9]. However, one can notice from (17) that either $\lambda_{1}=0$ or $R_{1}\left(\kappa_{s_{1}}^{*}\right)=R_{\text {min }_{1}}$. Similarly, (18) implies that either $\lambda_{2}=0$ or $R_{2}\left(\kappa_{s_{1}}^{*}\right)=R_{m i n_{2}}$. That said, four possible cases exist, as follows.

- Case 1: $\lambda_{1}=0$ and $\lambda_{2}=0$ means that both QoS constraints of user 1 and user 2 are inactive.

- Case 2: $\lambda_{1}=0$ and $\lambda_{2} \neq 0$ implies that the sub-optimal circularity coefficient exists when $R_{2}\left(\kappa_{s_{1}}^{*}\right)=R_{\text {min }_{2}}$.

- Case 3: $\lambda_{1} \neq 0$ and $\lambda_{2}=0$ implies that the sub-optimal circularity coefficient exists when $R_{1}\left(\kappa_{s_{1}}^{*}\right)=R_{\text {min }_{1}}$.

- Case 4: $\lambda_{1} \neq 0$ and $\lambda_{2} \neq 0$ implies that if the problem is feasible, the sub-optimal circularity coefficient exists when both $R_{1}\left(\kappa_{s_{1}}^{*}\right)=R_{\text {min }_{1}}$ and $R_{2}\left(\kappa_{s_{1}}^{*}\right)=R_{\text {min }_{2}}$.

The proposed algorithm to solve the problem in (14) can be formally summarized as follows.

1) Input: $R_{\text {min }_{1}}, R_{\text {min }_{2}}, P_{1}, P_{2}, h_{1}, h_{2}, \sigma^{2}$, and $\beta$.

2) Set $\lambda_{1}=\lambda_{2}=0$. Calculate $\kappa_{s_{1}}$ from (23). Calculate $R_{1}$ and $R_{2}$ from (12) and (13), respectively.

3) if $R_{1} \geq R_{\text {min }_{1}}$ and $R_{2} \geq R_{\text {min }_{2}}$, then, the sub-optimal solution $\kappa_{s_{1}}^{*}$ is reached.

4) else if $R_{1}<R_{\min _{1}}$ and $R_{2} \geq R_{\text {min }_{1}}$, then, find nonnegative $\lambda_{1}$ from (24) such that $R_{1}\left(\kappa_{s_{1}}\right)=R_{\text {min }_{1}}$ and re-calculate $\kappa_{s_{1}^{*}}$ from (23). Repeat until convergence.

5) else if $R_{1} \geq R_{\text {min }_{1}}$ and $R_{2}<R_{\text {min }_{1}}$, then, find nonnegative $\lambda_{2}$ from (24) such that $R_{2}\left(\kappa_{s_{1}}\right)=R_{\min _{2}}$ and re-calculate $\kappa_{s_{1}^{*}}$ from (23). Repeat until convergence.
6) else $R_{1}<R_{\min _{1}}$ and $R_{2}<R_{\text {min }_{1}}$, then, find non-negative $\lambda_{1}$ and $\lambda_{2}$ from (24) if exists such that $R_{1}\left(\kappa_{s_{1}}\right)=R_{\min _{1}}$ and $R_{2}\left(\kappa_{s_{1}}\right)=R_{\text {min }_{2}}$ and recalculate $\kappa_{s_{1}^{*}}$ from (23). Repeat until convergence.

7) Output: $\kappa_{s_{1}^{*}}$.

\section{Simulation Results}

In this section, we simulated a downlink two-user served by a base station in a NOMA system employing IGS and compare its achieved sum rate $\left(R_{1}+R_{2}\right)$ to its counterpart of PGSbased NOMA systems. Unless otherwise mentioned, $R_{\min _{1}}=$ $R_{\text {min }_{2}}=1.2 \mathrm{bits} / \mathrm{sec} / \mathrm{Hz}, P_{1}=0.3 P_{T}$, and $P_{2}=0.7 P_{T}$.

In Fig. 1, the sum-rate is simulated versus $\mathrm{SNR}=\frac{P_{T}}{\sigma_{n}^{2}}$, where $P_{T}$ is the total transmit power, at different values of $\beta$. As can be seen, the IGS-based NOMA system outperforms PGS-based NOMA for all levels of imperfect SIC. In particular, as the SIC becomes worse, i.e., $\beta=0.4$, the sum-rate gain of using IGS increases over PGS NOMA. IGS also offers a good gain in the low SNR region as the effect of the imperfect SIC is significant on the users' rate. At high SNR, the PGSbased NOMA system approaches the sum-rate performance of the IGS-based NOMA system. In addition, we use exhaustive search method to find the optimal solution and compare it with the proposed KKT sub-optimal solution. The results show that there is a small performance gap between the optimal solution and proposed sup-optimal solution in terms of sum-rate at low SNR values and the gap tends to zero at high SNR values. It is worthy note that the proposed solution is far less complex than the optimal solution of the exhaustive search. The figure also shows that in case of perfect SIC, i.e., $\beta=0$, both schemes perform similarly.

In Fig. 2, the sum-rate vs SNR for different values of $P_{1}, P_{2}$ at $\beta=0.3$ is simulated for both IGS and PGS NOMA system. As the strong user gains more power, i.e., $P_{1}$ becomes larger, the sum-rate curves shift up and show higher sum-rate. One can notice from Fig. 2 that different power allocation ratios do not affect the gain of IGS over PGS based NOMA systems.

The effect of users' channel strength on the sum-rate for IGS-based NOMA performance is shown in Fig. 3. The sumrate is simulated for the case of $\sigma_{h_{1}}^{2}=\{1,2,3,5,7,9\} \sigma_{h_{2}}^{2}$ at $P_{1}=0.1 P_{T}, P_{2}=0.9 P_{T}$, and $\beta=0.3$. It is clear that as $\sigma_{h_{1}}^{2}$ increases, the sum-rate enhances, i.e., as the channel of the first user becomes stronger, its rate becomes higher. Meanwhile, the rate of the user with weak channel is maximized by the proposed approach through the IGS-NOMA concept.

In Fig. 4, we show the convergence of the proposed algorithm at different values of $\beta$. On average, the algorithm needs small number of iterations to converge and the number increases as $\beta$ increases. 


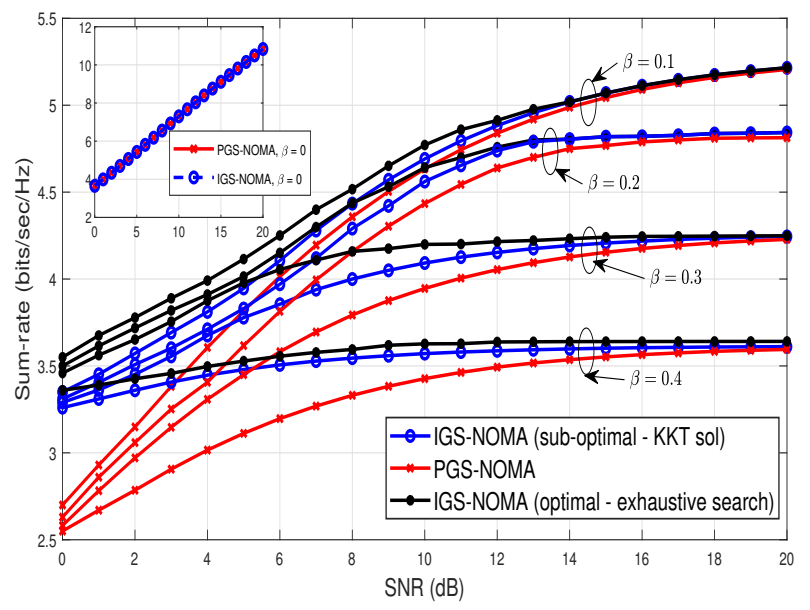

Fig. 1: Sum-rate vs SNR for IGS-based and PGS-based NOMA systems for different $\beta$.

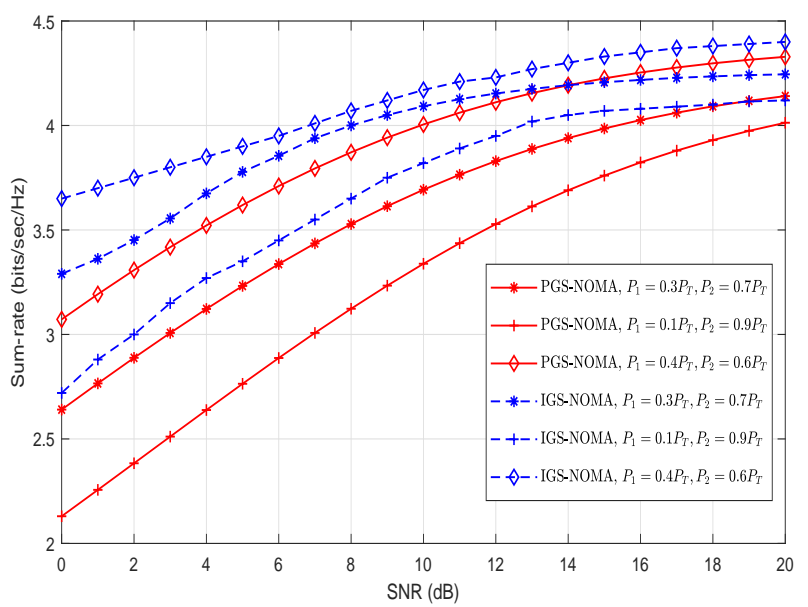

Fig. 2: Sum-rate vs SNR for IGS-based and PGS-based NOMA systems for different $P_{1}, P_{2}$ values, with $\beta=0.3$.

\section{CONCLUSION}

In this work, we optimized the sum-rate of a two-user NOMA system subject to minimum QoS requirements under imperfect SIC. An iterative algorithm was developed to find the sub-optimal IGS circularity coefficient that maximizes the sum-rate. Simulation results showed that IGS improves the sum-rate at low-to-medium SNR region. In addition, it was observed that the gain from IGS increases when imperfect SIC gets higher. Results also revealed that the power allocation does not affect the gain of IGS-based NOMA systems over its PGS-based counterpart. Moreover, sum-rate increases when channel gain ratio between users increases, but this improvement saturates at high SNR values.

\section{REFERENCES}

[1] M. Zeng, A. Yadav, O. A. Dobre, G. I. Tsiropoulos, and H. V. Poor, "Capacity comparison between MIMO-NOMA and MIMO-OMA with multiple users in a cluster," IEEE Journal Sel. Areas in Commun., vol. 35, no. 10, pp. 2413-2424, Oct. 2017.

[2] Y. Liu, Z. Qin, M. Elkashlan, A. Nallanathan, and J. A. McCann, "Nonorthogonal multiple access in large-scale heterogeneous networks," IEEE Journal Sel. Areas in Commun., vol. 35, no. 12, pp. 2667-2680, Dec. 2017.

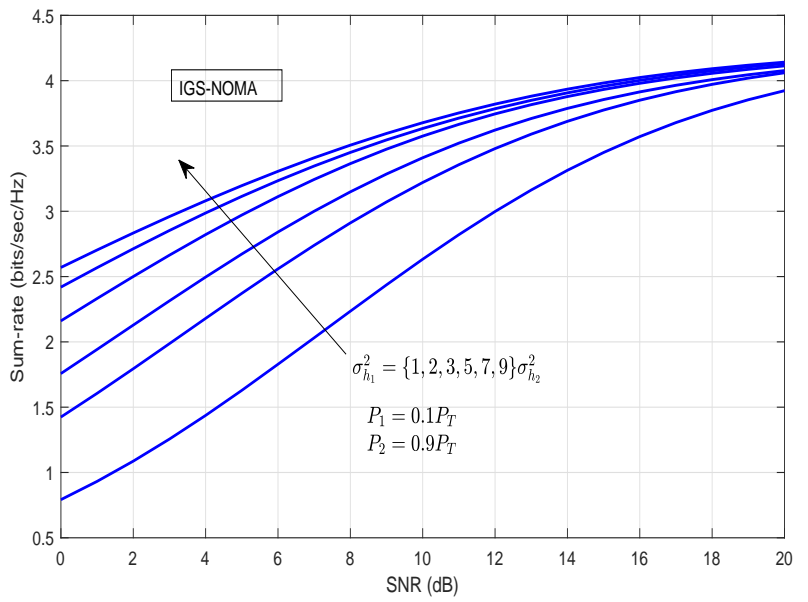

Fig. 3: Sum-rate vs SNR for IGS-based NOMA system for different $\sigma_{h_{1}}^{2}$ to $\sigma_{h_{2}}^{2}$ ratios, with $\beta=0.3, P_{1}=0.1 P_{T}$, and $P_{2}=0.9 P_{T}$.

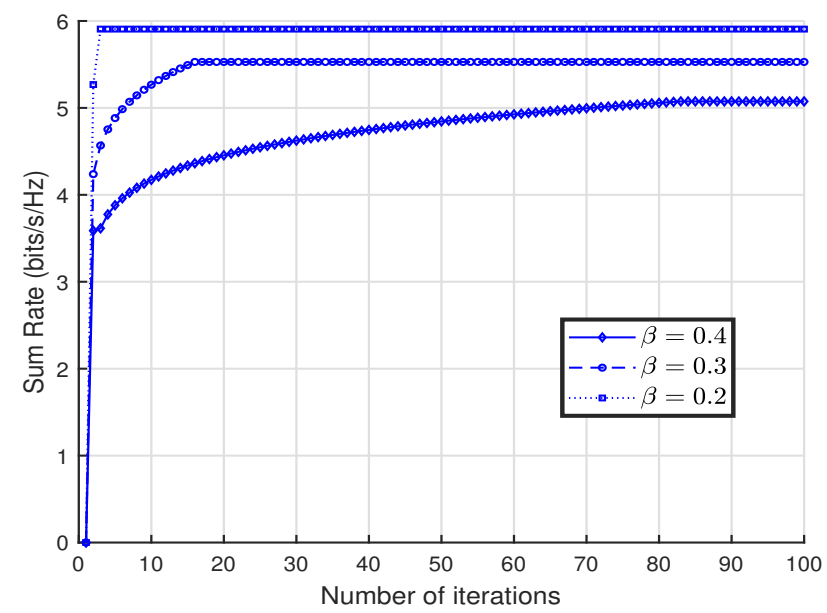

Fig. 4: Sum-rate vs number of iterations for algorithm convergence with $\beta=0.2,0.3,0.4$.

[3] L. P. Qian, Y. Wu, H. Zhou, and X. Shen, "Joint uplink base station association and power control for small-cell networks with non-orthogonal multiple access," IEEE Trans. Wireless Commun., vol. 16, no. 9, pp. 5567-5582, Sept. 2017.

[4] Y. Sun, D. W. K. Ng, Z. Ding, and R. Schober, "Optimal joint power and subcarrier allocation for full-duplex multicarrier non-orthogonal multiple access systems," IEEE Trans. Commun., vol. 65, no. 99, pp. 1077-1091, Mar. 2017.

[5] X. Yue, Z. Qin, Y. Liu, S. Kang, and Y. Chen, "A unified framework for non-orthogonal multiple access," to appear, IEEE Trans. Commun., vol. 66, no. 11, pp. 5346-5359, Nov. 2018.

[6] Y. Zeng, C. M. Yetis, E. Gunawan, Y. L. Guan, and R. Zhang, "Transmit optimization with improper Gaussian signaling for interference channels," IEEE Trans. Sig. Process., vol. 61, no. 11, pp. 2899-2913, Jun. 2013.

[7] O. Amin, W. Abediseid, and M. Alouini, "Overlay spectrum sharing using improper Gaussian signaling," IEEE Journal Sel. Areas in Commun., vol. 35, no. 1, pp. 50-62, Jan. 2017.

[8] F. D. Neeser and J. L. Massey, "Proper complex random processes with applications to information theory," IEEE Trans. Inf. Theory, vol. 39, no. 4, pp. 1293-1302, Jul. 1993.

[9] S. Boyd, L. Xiao, and A. Mutapcic, "Subgradient methods," Lecture Notes of EE392o, Stanford University, Autumn Quarter, 2003. 